Conclusion Children on HETF in our study maintain and had improvement of their micronutrient status after start of HETF. Six monthly blood test monitoring may only be necessary in special circumstances (refeeding syndrome, frankly deficient or toxic micronutrient levels at start of HETF) and may not significantly enhance the overall nutritional monitoring and management of all children on HETF.

Competing interests None declared.

\section{DDF plenary session OC-041 IL28B HAPLOTYPES AND IP-10 PREDICT TREATMENT RESPONSE FOR RECURRENT HCV POST TRANSPLANT}

doi:10.1136/gutjnl-2012-302514a.41

${ }^{1} \mathrm{D}$ Joshi, ${ }^{*}{ }^{1} \mathrm{I}$ Carey, ${ }^{2} \mathrm{M}$ Bruce, ${ }^{1} \mathrm{~A}$ Barnabas, ${ }^{1} \mathrm{~S}$ Knighton, ${ }^{1} \mathrm{M}$ Heneghan, ${ }^{1} \mathrm{~V}$ Aluvihare, ${ }^{1}$ A Suddle, ${ }^{1} \mathrm{~N}$ Heaton, ${ }^{1} \mathrm{~J}$ O'Grady, ${ }^{1} \mathrm{~K}$ Agarwal. ${ }^{1}$ Institute of Liver Studies, King's College Hospital, London, UK; ${ }^{2}$ Institute of Studies, King's College Hospital, London, UK

Introduction Hepatitis C virus (HCV) recurrence post liver transplant (LT) is universal. Sustained virological response (SVR) rates post LT with pegylated interferon (PEG-IFN) and ribavirin (RIB) range between $26 \%$ and $50 \%$ and are associated with significant side effects. Single nucleotide polymorphisms (SNPs) rs12979860 near the IL28B gene predict response to treatment. Strong immune T helper type 1 responses towards HCV determine also play an integral role in the outcome of infection. Interferon $\gamma$ inducible protein 10 (IP-10) has been shown to correlate with treatment response in HCV mono-infection and HIV co-infected patients but limited data are available for patients in the post LT period. Our aim was to investigate whether SNPs near IL28B gene rs12979860 and pretreatment plasma levels of IP-10 can predict treatment response in patients with recurrent HCV post LT.

Methods Pre-treatment plasma samples were studied in HCV patients post LT. Plasma levels of IP-10 (pg/ml) was measured by ELISA. rs12979860 were tested by direct sequencing. All patients were treated with PEG-IFN $\alpha 2 \mathrm{a}$ and weight based RIB for a minimum of 48 weeks irrespective of genotype. Virological response was divided into SVR, null-response (NR) and responder relapse (RR). All results are presented as medians (range).

Results 41 patients (34 male) with recurrent HCV (49\% genotype one disease) were treated at a median time of 43 months (3-133) post LT. $71 \%$ of patients were maintained on tacrolimus monotherapy. Nine patients had been treated previously with PEG-IFN and RIB. Median baseline HCV viral load was 2.35E6 IU/ml. $78 \%$ of patients were commenced on a low accelerated dosage regimen (median dose PEG $135 \mu \mathrm{g}$, median dose RIB $800 \mathrm{mg}$ ). Rs12979860 haplotype CC was present in $24 \%$ ( $8 \times$ SVR, $2 \times \mathrm{RR})$, CT $59 \%$ $(10 \times \mathrm{SVR}, 9 \times \mathrm{NR}, 5 \times \mathrm{RR})$ and TT in $17 \%(1 \times \mathrm{SVR}, 6 \times \mathrm{NR}) . \mathrm{SVR}$ was achieved by 19 patients (46\%), 15 patients were NR (37\%) and 7 were RR (17\%). Baseline IP-10 levels correlated with serum AST $(r=0.48, p=0.003)$, ALT $(r=0.36, p=0.05)$, fibrosis score $(r=0.33$, $\mathrm{p}=0.04)$ and necro-inflammatory score $(\mathrm{r}=0.54, \mathrm{p}=0.001)$. IP-10 levels were lower in those who achieved a SVR (116 vs 490, $p<0.0001)$. IP-10 levels were higher in the NR group compared to the SVR and RR groups (545 vs 116 vs 320, $\mathrm{p}<0.0001$ ). AUROC analysis identified IP-10 to be a significant predictor of SVR (0.84, $0.71-0.97, \mathrm{p}<0.0001)$. CC haplotype and IP-10 $<154 \mathrm{pg} / \mathrm{ml}$ had a $100 \%$ PPV for SVR.

Conclusion Our data demonstrates that patients with a lower baseline IP-10 level are more likely to achieve a SVR. The IL28B CC haplotype in conjunction with a low IP-10 level predicts treatment success in recurrent HCV post LT.

Competing interests None declared.

\section{OC-042 THE BURDEN OF MALNUTRITION IN GENERAL PRACTICE}

doi:10.1136/gutjnl-2012-302514a.42

${ }^{1} \mathrm{P}$ McGurk, ${ }^{*} \mathrm{~A}$ Cawood, ${ }^{1} \mathrm{E}$ Walters, ${ }^{2,3} \mathrm{R}$ J Stratton, ${ }^{3} \mathrm{M}$ Elia. ${ }^{1}$ Nutrition and Dietetics, University Hospital Southampton NHS Trust, Southampton; ${ }^{2}$ Medical Affairs, Nutricia, Trowbridge; ${ }^{3}$ Institute of Human Nutrition, University of Southampton, Southampton, UK

Introduction Although most malnutrition exists in the community, there is a lack of information about its burden in General Practice (GP). The aim of this survey was to establish the prevalence of malnutrition in GP and its relationship to use of nutrition support, health outcomes and healthcare use (infections requiring antibiotics, frequency of wounds and GP visits). The dataset represents an extension of information obtained from a preliminary study. ${ }^{1}$

Methods Of a total of 1130 consecutive individuals attending nine GPs in the Southampton area (November 2010-December 2011), $65 \%(n 738)$ agreed to participate (main reason for non-participation was fear of missing their appointment). After excluding 160 people who were accompanying friends/relatives or carers, 578 patients visiting the GP or nurse formed the survey population. Subjects had their height and weight measured and provided information about unintentional weight loss, infections requiring antibiotics, wounds, GP visits, dietetic input, and use of any form of oral nutritional support, during the preceding 6 months. The risk of malnutrition according to the "Malnutrition Universal Screening Tool" ("MUST") was established.

Results Patients had a mean age, weight and Body Mass Index (BMI) of $43.1(\mathrm{SD} \pm 18.7)$ years, $73.6(\mathrm{SD} \pm 17.0) \mathrm{kg}$, and 26.2 $(\mathrm{SD} \pm 5.3) \mathrm{kg} / \mathrm{m}^{2}$ respectively. The overall prevalence of malnutrition was $11.1 \%$ (95\% CI $8.8 \%$ to $13.9 \%$ ), comprising of $6.7 \%$ at medium risk and $4.4 \%$ at high risk. Compared to people at low risk of malnutrition, those "at risk" (medium + high risk) had significantly more infections requiring antibiotics $(17.1 \%$ vs $28.1 \%$, RR 1.643 (95\% CI 1.063 to 2.539); $\mathrm{p}=0.025$ ), a significantly higher frequency of wounds ( $2.1 \%$ vs $9.4 \%$, RR 4.381 (95\%CI 1.667 to 11.443 ); $\mathrm{p}=0.003)$ and more GP visits in the previous 6 months $(59.3 \%$ vs $68.8 \%$, RR 1.159 (95\% CI 0.968 to 1.387 ); $p=0.109$ ). None of the subjects identified as "at risk" of malnutrition were receiving dietetic input or any form of oral nutritional support.

Conclusion This survey has established that the prevalence of malnutrition among people visiting their GP in the Southampton area is $11.1 \%$ (95\% CI $8.8 \%$ to $13.9 \%$ ). The results indicate that those "at risk" of malnutrition have more infections, wounds, and tend to visit their GP more frequently. Furthermore, malnutrition is under-detected and under-treated in this setting.

Competing interests None declared.

\section{REFERENCE}

1. McGurk P, Cawood A, Walters E, et al. Proceedings of the Nutrition Society. 2012. In press.

\section{OC-043 CONTROLLED TRIAL OF IMMUNOABLATION AND AUTOLOGOUS HAEMOPOETIC STEM CELL TRANSPLANTATION IN CROHN'S DISEASE: INTERIM REPORT ON BEHALF OF THE ASTIC TRIALISTS}

doi:10.1136/gutjnl-2012-302514a.43

C Hawkey. ${ }^{*}$ Nottingham Digestive Diseases Centre \& BRU, University Hospital NHS Trust OMC Campus, Nottingham, UK

Introduction The Autologous Stem Cell Transplantation International Crohn's Disease (ASTIC) Trial is a randomised controlled evaluation of the proposition that immunoablation and hemopoietic stem cell transplantation improves the course of Crohn's disease. 
Recruitment of all 48 patients in the trial will complete in early 2012 and results to date are presented descriptively here.

Methods Patients with impaired quality of life due to active Crohn's disease, despite at least three immunosuppressive agents all receive mobilisation treatment (intravenous cyclophosphamide $4 \mathrm{gm} / \mathrm{m}^{2}$ over 2 days followed by recombinant human granulocyte-colony stimulating factor [GCSF, filgrastim], $10 \mu / \mathrm{kg}$ daily before randomisation to immediate (1 month) or delayed (1 year) immunoablation and stem cell transplantation. The conditioning regime is intravenous cyclophosphamide $50 \mathrm{mg} / \mathrm{kg}$ per day for 4 days, antithymocyte globulin $2.5 \mathrm{mg} / \mathrm{kg} /$ day and methyl prednisolone $1 \mathrm{mg} /$ $\mathrm{kg}$ on days $3-5$. The bone marrow is reconstituted by infusion of an unselected graft of $3-8 \times 106 / \mathrm{kg}$ CD34 positive stem cells. Results are compared 1 year after mobilisation alone or after transplantation.

Results Twelve months after stem cell transplantation (early or delayed) the Crohn's Disease Activity Index (CDAI) fell from 324 (median, IOR 229-411) to $161(85-257, \mathrm{n}=17)$ compared to 351 (287-443) to 272 (214-331) following mobilisation alone $(n=11)$. Six patients had a normal CDAI after transplantation vs one after mobilisation. C reactive protein fell from $16.6(6.7-32.0) \mathrm{mg} / \mathrm{l}$ to 6.5 $(3.5-12.5) \mathrm{mg} / \mathrm{l}$ vs $14(8.0-27.0) \mathrm{mg} / \mathrm{l}$ to 9.0 (2.0-23.4) mg/l following mobilisation alone. The Crohn's Disease Endoscopic Index of Severity (CDEIS) (aggregate for upper and lower endoscopy) fell from $18(10-25)$ to $5(1-11)$ following transplantation vs 14 (12-16) to 9 (4-22) following mobilisation. Three patients achieved the goal of a normal CDAI, no drug therapy and normal upper and lower endoscopy 1 year after tranplantation but so did one patient following mobilisation alone. Serious Adverse Events were common ( $n=100$ to date) with 42 infective episodes requiring or prolonging hospitalisation, following both mobilisation and conditioning and transplantation. There were seven episodes of viral (re)activation. Temporary flare of Crohn's disease activity or a need for surgery occurred in eight patients.

Conclusion Immunoablation and hemopoietic stem cell transplantation appears to be an effective treatment for some patients with Crohn's Disease, although full results will be required for a firm conclusion. Risks are significant, making it potentially suitable for only a limited number of patients. Data from the whole trial will be needed to judge whether mobilisation alone has any benefits.

Competing interests None declared.

\section{BSG endoscopy section free papers OC-044 GASTROSCOPY RATE IN ENGLISH GENERAL PRACTICE POPULATIONS: ASSOCIATION WITH OUTCOME FOR OESOPHAGOGASTRIC CANCER}

doi:10.1136/gutjnl-2012-302514a.44

${ }^{1} \mathrm{M}$ Shawihdi, ${ }^{*}{ }^{1} \mathrm{G}$ Powell, ${ }^{2} \mathrm{~N}$ Stern, ${ }^{2} \mathrm{~N}$ Kapoor, ${ }^{2} \mathrm{R}$ Sturgess, ${ }^{1} \mathrm{E}$ Thompson, ${ }^{1} \mathrm{M}$ Pearson, ${ }^{3} \mathrm{~K}$ Bodger. ${ }^{1}$ Aintree Health Outcomes Partnership, University of Liverpool, Liverpool, UK; ${ }^{2}$ Digestive Diseases Centre, Aintree University Hospital, Liverpool, UK; ${ }^{3}$ Department of Gastroenterology, Institute of Translational Medicine, University of Liverpool, Liverpool, UK

Introduction Rates of gastroscopy vary between English general practice populations. The magnitude of this variation suggests a wide spectrum of clinical practice. Current guidelines focus on alarm symptoms as triggers for investigation but early symptoms of cancer are non-specific. This project aimed to determine whether overall gastroscopy rate in GP practice populations in England is associated with outcome of oesophagogastric cancer (OGC), as measured by rate of major surgical resection, emergency admission for cancer diagnosis and mortality.

Methods Analysis of Hospital Episode Statistics (HES, 2006-2008) linked to death registry and practice population data. Gastroscopy volume determined by extracting total diagnostic gastroscopy procedures and aggregated at GP practice level. OGC cases: Methods developed and validated (using local \& national audit) to identify new cases of OGC and then extract all hospital episodes in chronological order, flag key milestones (eg, diagnostic gastroscopy; emergency admission to hospital; major surgery) using relevant diagnostic and procedure codes. Entry criteria: General practices with $\geq 1$ new case of OGC and with a per capita gastroscopy rate within a valid reference range (0.4-4.0 per 1000 population). Practices grouped into tertiles (low, medium and high gastroscopy rate).

Results 20709 OGC cases from 5956 practices serving an adult population of 35.1 million. Characteristics of OGC cases matched the national audit findings. Cases registered with practices in lowest tertile of gastroscopy rate had lowest rate of surgery (14\% vs $16 \%$ vs $16 \% ; \mathrm{p}=0.028)$, highest rate of emergency admission ( $29 \%$ vs $27 \%$ vs $25 \% ; \mathrm{p}<0.01$ ), and highest mortality at 6 months ( $41 \%$ vs $40 \%$ vs $39 \% ; \mathrm{p}<0.01)$. After adjustment for age, sex, co-morbidity and deprivation quintile in logistic regression analysis, the rate of gastroscopy (low, medium or high) at the patient's general practice was an independent predictor of all three outcomes.

Conclusion There is $>10$-fold variation in the rate of gastroscopy among general practice populations in England. On average, OGC patients belonging to practices within the lowest tertile have poorer outcomes. These findings suggest that guidelines aimed at reducing the use of gastroscopy may adversely affect cancer outcomes.

Competing interests None declared.

\section{OC-045 RANDOMISED PROSPECTIVE TRIAL OF TRANSNASAL VS STANDARD UPPER DIAGNOSTIC ENDOSCOPY UNDER LOCAL ANAESTHETIC: INTERIM ANALYSIS OF ENDOSCOPY QUALITY, PATIENT ACCEPTABILITY AND TOLERABILITY}

doi:10.1136/gutjnl-2012-302514a.45

E G Alexandridis, ${ }^{*}$ K Trimble, P Hayes, J N Plevris. Centre for Liver and Digestive Disorders, Royal Infirmary of Edinburgh, Edinburgh, UK

Introduction Transnasal upper gastrointestinal endoscopy (TNE) using ultrathin endoscopes is considered less invasive, thus an attractive alternative, if not a first choice option, for diagnostic upper endoscopy. This is the first prospective, randomised study, in a UK population to assess tolerability, acceptability and quality of TNE, in comparison with standard upper endoscopy (SOGD) under local anaesthetic.

Methods We prospectively recruited up to date 125 patients [59 male/66 female] mean age 57 years. The Fujinon EG530N (5.9 mm) and EG530WR $(9.4 \mathrm{~mm})$ endoscopes were used. The endoscopist and all patients completed detailed questionnaires regarding tolerability, acceptance and quality of each endoscopy using standard visual analogue scales (VAS). Oxygen saturation $\left[\mathrm{SaO}_{2}\right]$, heart rate [HR] and systolic blood pressure [SBP] were recorded during procedure. SOGD group received $\mathrm{O}_{2} 2 \mathrm{lt} / \mathrm{min}$. Quality of biopsies was evaluated blindly by the reporting pathologists.

Results Trial interim analysis included 129 endoscopies in 125 patients $[\mathrm{TNE}=65, \mathrm{SOGD}=64]$. In all patients intubation of $\mathrm{D} 2$ was achieved. VAS scores for patient comfort (higher score=greater comfort) were significantly better in the TNE compared to SOGD group ( 7 vs 5.6, respectively, $\mathrm{p}=0.0013$ ). 40 patients had previous experience of standard endoscopy, and $22(55 \%)$ reported gagging as main reason of discomfort. 22/40 were randomised to TNE. 21 of these 22 patients [95.5\%] stated preference to transnasal endoscopy in the future. Gagging score (higher score=more gagging) was significantly less in the TNE compared with SOGD group (0.05 vs 3.22 respectively, p2 (98\% vs $98.3 \%$, respectively, $\mathrm{p}=0.22$ ). Only 2 